\title{
Glycollate Inhibition of Growth of Pseudomonas aeruginosa on Lactate Medium
}

\author{
By P. R. BROWN* AND RENÉE TATA \\ Department of Biochemistry, King's College London, Strand, London WC2R 2LS, UK
}

(Received 26 August 1986; revised 8 January 1987)

\begin{abstract}
Glycollate inhibited growth of Pseudomonas aeruginosa in media containing either pyruvate or lactate as carbon sources. Glycollamide, but not glyoxylate, showed similar effects. Spontaneous mutants (L/G strains) were isolated that were able to grow on lactate medium in the presence of glycollate: their growth in pyruvate medium was still inhibited by glycollate. Synthesis of membrane-bound $\mathrm{NAD}^{+}$-independent $\mathrm{D}(-)$ - and $\mathrm{L}(+)$-lactate dehydrogenases (iLDHs) was inducible by $D$ - or L-lactate in the parent strain but was constitutive in the $L / G$ strains. Glycollate inhibited induction of the synthesis of iLDHs in the parent strain growing in succinate medium but had no effect under the same conditions on strain L/G1. Glycollate was a competitive inhibitor of $\mathrm{L}(+)$-iLDH $\left(K_{\mathrm{i}}=11 \mathrm{mM}\right)$. No differences were found in the kinetic properties of $\mathrm{L}(+)-\mathrm{iLDH}$ in cell-free extracts from strain $\mathrm{L} / \mathrm{Gl}$ and the parent organism. Glycollate appears to inhibit growth on lactate medium predominantly through prevention of lactate induction of iLDH synthesis.
\end{abstract}

\section{INTRODUCTION}

The metabolism of DL-lactate by Pseudomonas aeruginosa (Kemp, 1972), Pseudomonas citronellis (O'Brien, 1977a) and Pseudomonas putida (O'Brien, 1977b) seems to depend on the induction of $\mathrm{NAD}^{+}$-independent $\mathrm{L}(+)$ and $\mathrm{D}(-)$-lactate dehydrogenases (iLDHs) (EC1.1.1.27 and EC1.1.1.28, respectively) (for a review of LDHs see Garvie, 1980) that catalyse the conversion of lactate to pyruvate. Kemp (1972) showed that in P. aeruginosa these enzymes were membrane-bound and interacted with the electron transport chain. The iLDHs of $P$. citronellis (O'Brien, $1977 a$; O'Brien and Taylor, 1977) and $P$. putida (O'Brien, 1977b) appear to be similar to those in $P$. aeruginosa although the pattern of induction differs slightly for the different species. In $P$. putida and $P$. aeruginosa either D-lactate or L-lactate induces synthesis of both enzymes but, in $P$. citronellis, L-lactate induces both iLDHs, whereas D-lactate induces only D( -)-iLDH.

With lactate as carbon source no growth of $P$. aeruginosa occurs if glycollamide is used as a nitrogen source though the organism synthesizes an aliphatic amidase catalysing the hydrolysis of glycollamide to give ammonia and glycollate (Brown \& Tata, 1987; this paper). In a taxonomic study of the aerobic pseudomonads, Stanier et al. (1966) reported that although a few Pseudomonas spp. are able to metabolize glycollate, $P$. aeruginosa strains cannot use it as a carbon source. It seemed possible therefore that glycollate prevented growth on lactate. In this paper we describe the results of experiments to confirm this and to elucidate the mechanism involved. A preliminary report of this work has appeared previously (Brown \& Tata, 1981).

\section{METHODS}

Bacterial strains. The strains used (obtained from NCIB, Aberdeen, UK) were Pseudomonas aeruginosa PAC 1, described originally as strain 8602 by Kelly \& Clarke (1962) and strain L10, a mutant derived from strain PAC 1

Abbreviations: iLDH, NAD+-independent lactate dehydrogenase; DCPIP, dichlorophenolindophenol; PMS, phenazine methosulphate. 
that harbours mutations affecting the regulation of synthesis of amidase (Brown \& Clarke, 1970; Smyth \& Clarke, 1975).

Growth media. Minimal media, containing as carbon sources sodium DL-lactate $(0.3 \%, \mathrm{w} / \mathrm{v})$, sodium pyruvate $(0.3 \%, \mathrm{w} / \mathrm{v})$, sodium acetate $(0.3 \%, \mathrm{w} / \mathrm{v})$ or potassium glycollate $(0.1 \%, \mathrm{w} / \mathrm{v})$, were prepared by adding the appropriate solution, sterilized by filtration, to autoclaved minimal salts medium (Brammar \& Clarke, 1964) containing $0.1 \%(\mathrm{w} / \mathrm{v})\left(\mathrm{NH}_{4}\right)_{2} \mathrm{SO}_{4}$ as nitrogen source. Succinate medium was prepared in a similar way but sodium succinate $(0 \cdot 3 \%, w / v)$ was autoclaved with the medium. Solid media contained $1 \%(w / v)$ Difco Noble Agar. Lactate/glycollate solid medium contained sodium DL-lactate $(0.05 \%, w / v)$ and potassium glycollate $(0.2 \%$, w/v).

Growth inhibition studies. The effects of glycollate and glycollamide on bacterial growth in liquid media were investigated by growing bacteria in $250 \mathrm{ml}$ culture medium at $37^{\circ} \mathrm{C}$ to early exponential phase $\left(\mathrm{OD}_{670}=0 \cdot 15\right)$ in 11 conical fiasks, and then transferring $45 \mathrm{ml}$ portions to $250 \mathrm{ml}$ flasks containing $5 \mathrm{ml}$ sterile glycollate or glycollamide solution. Flasks were shaken in a Gallenkamp orbital shaker at $37^{\circ} \mathrm{C}$ and samples removed at intervals to monitor growth by measuring $\mathrm{OD}_{670}$ in a Unicam SP600 spectrophotometer.

Isolation of $L / G$ mutants. A portion $(5 \mathrm{ml})$ of overnight broth culture of strain $\mathrm{L} 10$ was centrifuged and the bacteria were washed in $5 \mathrm{ml}$ dilution buffer (Brammar et al., 1967). After centrifugation, bacteria were resuspended in $5 \mathrm{ml}$ dilution buffer and portions $(0.1 \mathrm{ml})$ were spread on lactate/glycollate medium and incubated for $48 \mathrm{~h}$ at $37^{\circ} \mathrm{C}$. Mutant colonies were purified by restreaking on the isolation medium to obtain single colonies.

Enzyme assays. iLDHs were assayed using an adaptation of the method of Bennett et al. (1966). The assay mixture contained the following: dichlorophenolindophenol (DCPIP), $0.13 \mathrm{mM}$, phenazine methosulphate (PMS), $0.65 \mathrm{mM}$, and potassium cyanide, $0.4 \mathrm{mM}$, in $0.06 \mathrm{M}$-potassium phosphate buffer, $\mathrm{pH}$ 6.8. Bacterial extracts were added to $1 \mathrm{ml}$ of reaction mixture in cuvettes equilibrated at $37^{\circ} \mathrm{C}$ and the $A_{610}$ was monitored using a Unicam SP800 recording spectrophotometer for 2-3 min before starting the reaction by adding either lithium $\mathrm{L}$ lactate $(7.5 \mathrm{mM})$ or lithium D-lactate $(7.5 \mathrm{~mm})$. The reaction was monitored by measuring the rate of decrease in $A_{610}$. Enzyme activities are expressed as nmols DCPIP reduced $\mathrm{min}^{-1}$ (mg protein) ${ }^{-1}$ using a molar absorption coefficient $\varepsilon_{610}=1.9 \times 10^{4} \mathrm{Imol}^{-1} \mathrm{~cm}^{-1}$ for DCPIP.

Preparation of cell-free extracts. Bacteria were harvested by centrifugation, washed in dilution buffer and then resuspended in $1 / 100$ th of the original medium volume of $100 \mathrm{~mm}$-potassium phosphate buffer, $\mathrm{pH} 6 \cdot 8$, at $0{ }^{\circ} \mathrm{C}$. Cells were broken by sonication using an MSE Mullard ultrasonic disintegrator (power output $50 \mathrm{~W}$ ) for $2 \mathrm{~min}$ at $0{ }^{\circ} \mathrm{C}$. Cell debris was removed by centrifugation at $22000 \mathrm{~g}$ in an MSE Angle 18 centrifuge at $4{ }^{\circ} \mathrm{C}$ for $15 \mathrm{~min}$. Supernatant solutions were stored on ice before assay.

Protein estimations. These were done by the method of Lowry using bovine serum albumin as a standard.

Chemicals. Chemicals were of analytical grade. DCPIP and PMS were obtained from Sigma. Glycollic acid was recrystallized from ethanol twice and was neutralized with potassium carbonate before use in growth media. Glycollamide was prepared by the ammoniolysis of glycollic acid ethyl ester obtained from Eastman-Kodak (Schmuck, 1924) and was recrystallized twice from ethanol before use.

\section{RESULTS}

\section{Growth inhibition by glycollate}

Stanier $e$ t al. (1966) reported that glycollate is unable to support growth of $P$. aeruginosa. This was confirmed for strains PAC 1 and L10: neither strain produced visible colonies on solid minimal medium containing glycollate as sole carbon source even after 2-3 d incubation at $37^{\circ} \mathrm{C}$, and neither strain grew in liquid medium containing glycollate.

Plate tests showed that inclusion of $0.1 \%$ glycollate in pyruvate or lactate minimal media prevented growth of strains $\mathrm{L} 10$ and PAC 1 . On succinate medium the presence of glycollate made no apparent difference to colony size after overnight incubation. Glyoxylate, the immediate product of glycollate metabolism in glycollate-utilizing Pseudomonas spp. (Kornberg $\&$ Gotto, 1961) had no discernible effect at a concentration of $0.1 \%$ on growth on lactate or pyruvate media, which suggested that glycollate itself, rather than an intermediate in a blocked glycollate metabolic pathway, was the growth inhibitor.

Spontaneous mutants (L/G strains) were isolated from strain L10 on lactate/glycollate medium at a frequency of about 1 per $10^{6}$ cells plated. The growth properties of 10 of these mutants, picked at random, were examined on solid media. None grew on glycollate as sole carbon source but all were able to grow in the presence of glycollate plus lactate or glycollate plus succinate; none grew on pyruvate medium containing glycollate. The susceptibilities to glycollate inhibition of growth of the parent and one mutant strain, $L / G 1$, were compared in more detail in liquid media (Fig. 1): 52 mM-glycollate strongly repressed growth of strain L10 in lactate medium but had no effect on strain L/G1. 

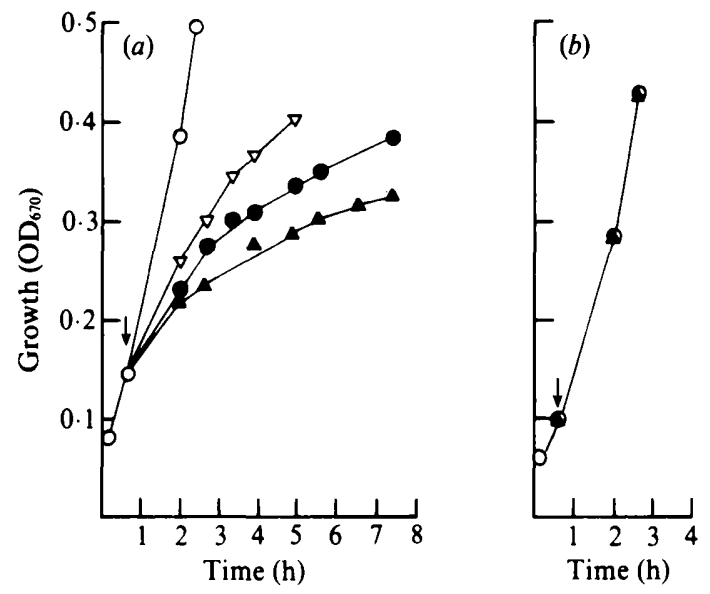

Fig. 1. Effects of glycollate on the growth of $P$. aeruginosa strains L10 (a) and L/G1 $(b)$ in lactate medium. Arrows indicate the time at which glycollate was added (see Methods). Glycollate concentrations were as follows: $0(O) ; 6.5 \mathrm{~mm}(\nabla) ; 13 \mathrm{~mm}(\bigcirc) ; 52.5 \mathrm{~mm}(\Lambda)$.
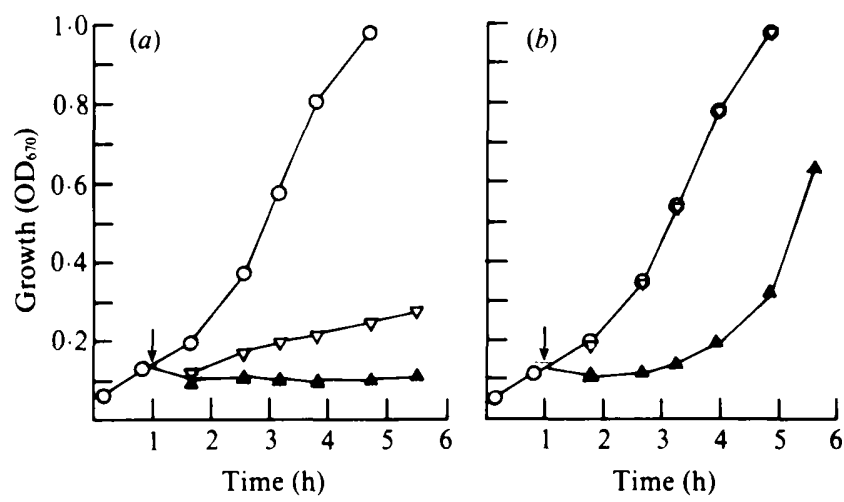

Fig. 2. Effects of glycollamide on the growth of $P$. aeruginosa strains L10 (a) and L/G1 $(b)$ in lactate medium. Glycollamide was added (see Methods) at the time indicated by the arrows. Concentrations of glycollamide were as follows: $0(\bigcirc) ; 13.3 \mathrm{~mm}(\nabla) ; 33 \mathrm{~mm}(\boldsymbol{\Delta})$.

To examine the possibility that the mutation in strain L/G1 reduced the susceptibility to glycollate inhibition by preventing glycollate uptake, the effects of glycollamide on growth of the parent and mutant strains in lactate medium were compared. Farin (1977) concluded that $P$. aeruginosa PAC 1 is freely permeable to aliphatic amides and that amide uptake depends on the activity of an aliphatic amidase that catalyses the hydrolysis of the amide and includes glycollamide within its range of substrates (Kelly \& Clarke, 1962). Glycollamide at the same concentration had a more pronounced effect than glycollate on strain L10. The mutant L/G1 under the same growth conditions was less susceptible to inhibition by glycollamide although some residual susceptibility remained: $33 \mathrm{~mm}$-glycollamide completely suppressed growth of strain L10 and initially slowed growth of strain L/G1 (Fig. 2). Parent and mutant strains had the same level of amidase activity (data not shown) so the observation indicated that the relative resistance of the mutant strain to glycollate inhibition was not due to impermeability to glycollate.

In pyruvate medium no differences were observed between strains $\mathrm{L} 10$ and $\mathrm{L} / \mathrm{G} 1$ in their susceptibilities to growth inhibition either by glycollate or glycollamide. Concentrations of glycollamide and glycollate that suppressed growth completely ( $8 \cdot 3$ and $26 \mathrm{~mm}$ respectively) were lower than those required to prevent growth of strain L10 in lactate medium. No effects on 
Table 1. iLDH activities in P. aeruginosa strains $L 10$ and $L / G 1$ grown in various media

Activities are expressed as $\mathrm{nmol}$ lactate oxidized $\mathrm{min}^{-1}(\mathrm{mg} \text { protein })^{-1}$ with DCPIP as electron acceptor. Assays were done on exponential phase cultures except on those grown in succinate and acetate media when stationary phase cells were used. Activities represent means of duplicate assays done in two separate experiments. ND, Not determined.

\begin{tabular}{|c|c|c|c|c|}
\hline \multirow{2}{*}{$\begin{array}{l}\text { Carbon } \\
\text { source for } \\
\text { growth }\end{array}$} & \multicolumn{2}{|c|}{ Strain L10 } & \multicolumn{2}{|c|}{ Strain $\mathrm{L} / \mathrm{G} 1$} \\
\hline & $\underset{\text { activity }}{\mathrm{L}(+)-\mathrm{iLDH}}$ & $\begin{array}{c}\mathrm{D}(-)-\mathrm{iLDH} \\
\text { activity }\end{array}$ & $\begin{array}{l}\mathrm{L}(+)-\mathrm{iLDH} \\
\text { activity }\end{array}$ & $\begin{array}{l}\mathrm{D}(-) \text {-iLDH } \\
\text { activity }\end{array}$ \\
\hline Succinate & 4 & ND & 96 & ND \\
\hline Acetate & 9 & ND & 368 & ND \\
\hline Pyruvate & 9 & 0 & 211 & 224 \\
\hline DL-Lactate & 210 & 77 & 480 & 252 \\
\hline L-Lactate & 310 & 207 & ND & ND \\
\hline
\end{tabular}

growth in succinate medium were observed for either strain with glycollate concentrations up to $19.7 \mathrm{mM}$ and glyoxylate $(16 \mathrm{mM})$ had no effect on growth in lactate medium confirming the observations made on solid media.

\section{Synthesis of iLDHs}

iLDH activities were measured in extracts of strain L10 grown in a variety of media. iLDH activity was found in cells grown in the presence of lactate but only very low levels were detectable in the absence of lactate (Table 1). D-Lactate ( $3 \mathrm{mM})$ and L-lactate (1-13 mM) were tested separately as inducers of iLDH synthesis in cells of strain L10 growing in succinate medium: both resulted in synthesis of $L(+)$-iLDH and $D(-)$-iLDH. Variations in the concentration of L-lactate had no effect on the level of iLDH (data not shown).

In striking contrast to the parent strain, synthesis of iLDHs in all the $\mathrm{L} / \mathrm{G}$ mutants occurred both in the presence and in the absence of lactate. Data are shown in Table 1 only for strain $\mathrm{L} / \mathrm{G} 1$. For several, though not all, of the $\mathrm{L} / \mathrm{G}$ strains tested, iLDH activities of cells grown in lactate medium were higher than those for strain $\mathrm{L} / \mathrm{Gl}$. iLDH activities were lowest in cells grown in succinate medium but were still at least 20 -fold higher in the $\mathrm{L} / \mathrm{G}$ strains than in strain L10.

The apparently constitutive synthesis of both lactate dehydrogenases in the $\mathrm{L} / \mathrm{G}$ mutant strains suggested that glycollate might normally exert its inhibitory effect on growth on lactate medium by repressing induction of lactate dehydrogenases. To test this possibility, the effect of glycollate on induction by lactate of iLDHs in strains L10 and L/G1 growing in succinate medium was tested. Succinate was chosen because glycollate exhibited no detectable growth inhibitory effects in succinate medium. The results, given in Table 2, showed that for strain L10, as the glycollate concentration in the medium was increased so the level of iLDHs induced by lactate was reduced. In strain $\mathrm{L} / \mathrm{G} 1$, in contrast, glycollate at a concentration that caused complete inhibition of induction of iLDHs in the parent showed no effect on iLDH synthesis.

\section{Inhibition of iLDHs by glycollate}

The structural similarities between lactate and glycollate suggested that glycollate might act as an inhibitor of iLDH activity; this could therefore be a contributory factor in growth inhibition by glycollate. Preliminary experiments using cell extracts as sources of enzyme showed that glycollate inhibited both $\mathrm{D}(-)-\mathrm{iLDH}$ and $\mathrm{L}(+)$-iLDH. The effects of varying the concentration of glycollate on $\mathrm{L}(+)$-iLDH activity at different fixed concentrations of L-lactate were investigated and values for $K_{\mathrm{i}}$, determined using a Dixon plot (not shown), were $12.5 \mathrm{~mm}$ for strain L10 and $11 \mathrm{~mm}$ for strain L/G1. Replots (not shown) of the data by the method of CornishBowden (1974) gave parallel lines, indicating competitive inhibition. Determinations of the $K_{\mathrm{m}}$ of $\mathrm{L}(+)$-iLDH for $\mathrm{L}$-lactate using cell free extracts gave linear double reciprocal plots and values of $0.25 \mathrm{~mm}$ for strain $\mathrm{L} 10$ and $0.27 \mathrm{~mm}$ for strain $\mathrm{L} / \mathrm{G} 1$. 
Table 2. Effect of glycollate on induction of $\mathrm{L}(+)-i L D H$ synthesis in P. aeruginosa cells growing in succinate medium

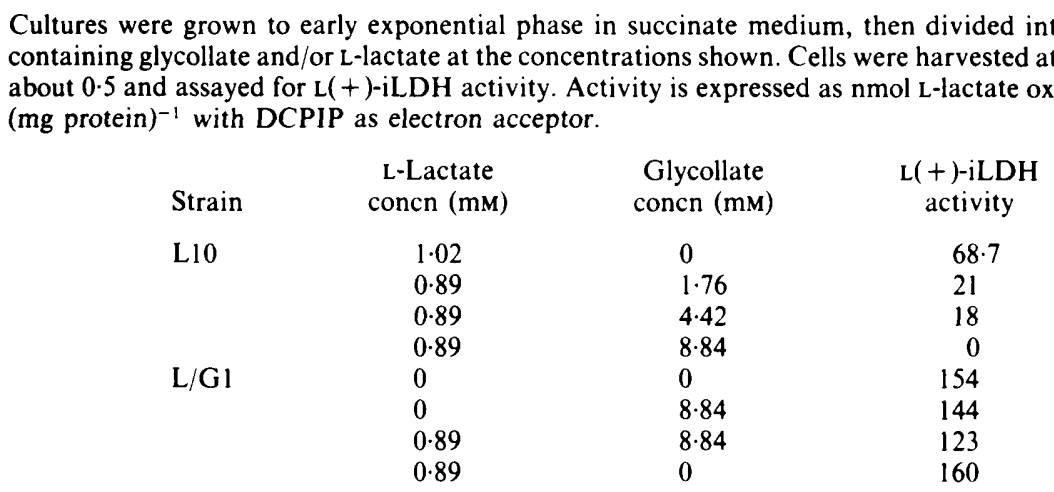

DISCUSSION

The properties of the $\mathrm{L} / \mathrm{G}$ mutants indicate that a reduction in the sensitivity of $P$. aeruginosa to inhibition of growth by glycollate in lactate medium is associated with mutations resulting in constitutive synthesis of iLDHs. This suggested that the primary action of glycollate is through repression of $\mathrm{iLDH}$ induction, and evidence is presented showing that glycollate normally inhibits induction of iLDH synthesis by lactate. The mechanism of this proposed inhibition is not clear. It could be caused through competition of glycollate with lactate for entry into the cell. Matin \& Konings (1973) showed that uptake rates of lactate into cells of a freshwater Pseudomonas (species unidentified) were inhibited nearly $60 \%$ by $1 \mathrm{~mm}$-glycollate. However, it seems unlikely that iLDHs themselves are directly involved in lactate uptake since entry of DLlactate into membrane vesicles is not dependent on iLDH (Matin \& Konings, 1973), and O'Brien $(1977 b)$ concluded that uptake of D- and L-lactic acids was independent of iLDH activity in $P$. putida. It is feasible that synthesis of a lactate permease could be coordinately regulated with transcription of iLDH genes and constitutive synthesis would allow lactate entry in the presence of glycollate by providing more binding sites. Alternatively, glycollate inhibition of iLDH induction may be an intracellular event, glycollate competing with lactate for binding to a hypothetical regulatory protein that controls transcription of $i \mathrm{LDH}$ genes. We have screened several hundred $\mathrm{L} / \mathrm{G}$ mutants to identify any that are able to grow on the selection medium at $40{ }^{\circ} \mathrm{C}$ but not at $25^{\circ} \mathrm{C}$, but without success (C. L. Crossley \& P. R. Brown, unpublished), which argues against the presence of a regulatory protein requiring lactate binding to activate transcription.

The inhibitory effects of glycollamide on growth with lactate depend on its conversion to glycollate since amidase-negative mutants show no such susceptibility (Brown \& Tata, 1987), but this does not necessarily imply that glycollate inhibition is exerted intracellularly since glycollate might be exported after formation from glycollamide then taken up by an active transport process as Farin (1977) has suggested for acetate formed from acetamide. Using $\left[{ }^{14} \mathrm{C}\right]$ glycollate we have found that the label is taken up into the cell by an azide-sensitive process (A. Allsop \& P. R. Brown, unpublished).

No large differences were found between the kinetic parameters of the iLDHs of parent and mutant strains, suggesting that the differences in enzyme activities are unlikely to be due to structural changes in the enzymes. The inhibitory action of glycollate on enzyme activity is relatively weak, reflected in a high $K_{\mathrm{i}}$ value, but could contribute to growth inhibition depending on the relative intracellular concentrations of glycollate and lactate.

The continued sensitivity of $\mathrm{L} / \mathrm{G}$ mutants to glycollate inhibition of growth on pyruvate medium seems to indicate an effect of glycollate on transport of pyruvate into the cell, unless pyruvate formed by iLDH activity towards lactate is somehow differentiated by, for example, channelling or chemical modification, from pyruvate entering the cell. 
The induction of both iLDHs by either D- or L-lactate suggests that the enzymes are coordinately regulated although the possibility of a racemase interconverting the two isomers has not been eliminated. Since regulation of both enzymes was similarly affected in the $L / G$ strains [and in $\mathrm{L} / \mathrm{G}$ mutants isolated on L-lactate/glycollate medium (P. R. Brown, unpublished], this too strongly indicates that the synthesis of the enzymes is coordinately controlled. The high frequency at which $L / G$ mutants arise makes unlikely the possibility that each is the result of more than one mutation.

\section{REFERENCES}

Bennett, R., Taylor, D. R. \& Hurst, A. (1966). Dand L-lactate dehydrogenases in Escherichia coli. Biochimica et biophysica acta 118, 512-521.

Brammar, W. J. \& Clarke, P. H. (1964). Induction and repression of Pseudomonas aeruginosa amidase. Journal of General Microbiology 37, 307-319.

Brammar, W. J., Clarke, P. H. \& Skinner, A. J. (1967). Biochemical and genetic studies with regulator mutants of the Pseudomonas aeruginosa 8602 amidase system. Journal of General Microbiology 47 , 87-102.

Brown, J. E. \& Clarke, P. H. (1970). Mutations in a regulator gene allowing Pseudomonas aeruginosa 8602 to grow in butyramide. Journal of General Microbiology 64, 329-342.

Brown, P. R. \& TATA, R. (1981). Mutants of Pseudomonas aeruginosa resistant to growth inhibition by glycollamide. Biochemical Society Transactions $9(2), 306 \mathrm{P}$.

BROWN, P. R. \& TATA, R. (1987). Isolation of amidasenegative mutants of Pseudomonas aeruginosa using glycollamide as a selective agent. Journal of General Microbiology 133, 1527-1533.

CORNISH-BOWDEN, A. (1974). A simple graphical method for determining the inhibition constants of mixed, uncompetitive and non-competitive inhibitors. Biochemical Journal 137, 143-144.

FARIN, F. (1977). Studies on the mechanism of regulation of amidase synthesis in Pseudomonas aeruginosa. $\mathrm{PhD}$ thesis, University of London, UK.

GAR VIE, E. I. (1980). Bacterial lactate dehydrogenases. Microbiological Reviews 44, 106-139.

Kelly, M. \& Clarke, P. H. (1962). An inducible amidase produced by a strain of Pseudomonas aeruginosa. Journal of General Microbiology 27, 305316.

KEMP, M. B. (1972). D- and L-lactate dehydrogenases of Pseudomonas aeruginosa. Biochemical Journal 130 , 307-309.

KORNBERG, H. L. \& GotTo, A. M. (1961). The metabolism of $\mathrm{C}_{2}$ compounds in micro-organisms and synthesis of cell constituents from glycollate by Pseudomonas sp. Biochemical Journal 78, 69-82.

Matin, A. \& Konings, W. N. (1973). Transport of lactate and succinate by membrane vesicles of Escherichia coli, Bacillus subtilis and a Pseudomonas species. European Journal of Biochemistry $34,58-67$.

O'BrIEN, R. W. (1977a). Induction of NAD-independent D-lactate dehydrogenase in Pseudomonas citronellis. FEMS Microbiology Letters 1, 343-346.

O'BRIEN, R. W. (1977b). Metabolism of D- and Llactate by $P$ seudomonas putida. Australian Journal of Biological Sciences 30, 553-558.

O'Brien, R. W. \& TAYLOR, B. L. (1977). Formation and dissimilation of oxaloacetate and pyruvate in Pseudomonas citronellis grown on non-carbohydrate sources. Journal of Bacteriology 130, 131-135.

Schmuck, A. (1924). Die Verkettungsreaktion der Amide von $\alpha$-oxysaüren. Biochemische Zeitschrift 147, 193-202.

Smyth, P. F. \& Clarke, P. H. (1975). Catabolite repression of Pseudomonas aeruginosa amidase: the effects of carbon source on amidase synthesis. Journal of General Microbiology 90, 81-90.

Stanier, R. Y., Palleroni, N. J. \& Doudoroff, M. (1966). The aerobic Pseudomonads: a taxonomic study. Journal of General Microbiology 47, 159-271. 